\title{
NECESSARY AND SUFFICIENT CONDITIONS FOR THE SOLVABILITY OF THE INVERSE PROBLEM FOR NON-SELF-ADJOINT PENCILS OF STURM-LIOUVILLE OPERATORS ON THE HALF-LINE
}

\author{
V. A. YURKO
}

\begin{abstract}
Non-self-adjoint Sturm-Liouville differential operators on the half-line with a boundary condition depending polynomially on the spectral parameter are studied. We investigate the inverse problem of recovering the operator from the Weyl function. For this inverse problem we provide necessary and sufficient conditions for its solvability along with a procedure for constructing its solution by the method of spectral mappings.
\end{abstract}

\section{Introduction}

Consider the differential equation and the boundary condition $L=L(q, U)$ :

$$
\begin{aligned}
\ell y & :=-y^{\prime \prime}+q(x) y=\lambda y, \quad x>0, \\
U(y) & :=P_{1}(\lambda) y^{\prime}(0)-P_{0}(\lambda) y(0)=0,
\end{aligned}
$$

where $\lambda$ is the spectral parameter, $q(x) \in L(0, \infty)$ is a complex-valued function, which is called the potential, and

$$
P_{k}(\lambda)=\sum_{j=0}^{p_{k}} P_{k j} \lambda^{p_{k}-j}, \quad k=0,1, \quad p_{k} \geq 0,
$$

are polynomials of degree $p_{k}$ with complex coefficients such that $P_{1}(\lambda)$ and $P_{0}(\lambda)$ has no common zeros.

In this paper, we study an inverse spectral problem for $L$. Inverse problems of spectral analysis consist in recovering operators from their spectral characteristics and arise in many fields. In particular, several examples of spectral problems arising in mechanical engineering and having boundary conditions depending on the spectral parameter are provided in the book [1] of Collatz; see also [2, 3, 4], where further references and links to applications can be found.

2000 Mathematics Subject Classification. 34A55 34B24 47E05.

Key words and phrases. Sturm-Liouville operators, boundary conditions with the spectral parameter, inverse spectral problems, method of spectral mappings. 
Classical inverse problems for Sturm-Liouville operators without $\lambda$ in boundary conditions have been studied fairly completely (see the monographs [5]-[10] and the references therein). Inverse problems for differential operators with boundary conditions dependent on the spectral parameter are more difficult to investigate, and nowadays there are only a number of papers in this direction (see [11]-[17]). In particular, [11]-[16] study such problems on a finite interval. Inverse spectral problems for the non-self-adjoint Sturm-Liouville pencil (1)(2) on the half-line were considered in [17] where the inverse problem of recovering $L$ from the Weyl function was studied and a constructive solution of this inverse problem was provided.

In this paper we consider singular non-self-adjoint Sturm-Liouville pencils of the form (1)-(2) on the half-line. We provide necessary and sufficient conditions for the solvability of the inverse problem of recovering $L$ from the Weyl function. The obtained results are natural generalizations of the well-known results on the classical inverse problems.

Let for definiteness, $p_{1}=p_{0}$. Other cases can be treated similarly. Without loss of generality we put $P_{10}=1$. Denote by $Z_{k}=\left\{z_{k s}\right\}_{s=\overline{1, p_{k}}}$ the zeros of $P_{k}(\lambda), k=0,1$. Then

$$
P_{k}(\lambda)=P_{k 0} \prod_{s=1}^{p_{k}}\left(\lambda-z_{k s}\right), \quad k=0,1
$$

Let $\lambda=\rho^{2}$, and let for definiteness $\operatorname{Im} \rho \geq 0$. Denote by $\Pi_{+}$the $\lambda$-plane with the two-sided cut $\Pi_{0}$ along the arc $\Lambda_{+}:=\{\lambda: \lambda \geq 0\}$, and $\Pi:=\overline{\Pi_{+}} \backslash\{0\}$; notice that here $\Pi_{+}, \Pi_{0}$ and $\Pi$ must be considered as images of subsets of the Riemann surface of the squareroot-function. Then, under the map $\rho \rightarrow \rho^{2}=\lambda, \Pi_{+}, \Pi_{0}$ and $\Pi$ correspond to the domains $\Omega_{+}=\{\rho: \operatorname{Im} \rho>0\}, \Omega_{0}=$ $\{\rho: \operatorname{Im} \rho=0\}$ and $\Omega=\{\rho: \operatorname{Im} \rho \geq 0, \rho \neq 0\}$, respectively.

Let $\Phi(x, \lambda)$ be the solution of equation (1) under the conditions $U(\Phi)=1, \quad \Phi(x, \lambda)=$ $O(\exp (i \rho x)), x \rightarrow \infty, \rho \in \Omega_{+}$, where $U$ is defined by (2). Denote $M(\lambda):=\Phi(0, \lambda)$. We will call $M(\lambda)$ the Weyl function for $L$, since it is a generalization of the concept of the Weyl function for the classical Sturm-Liouville operator (see [8]).

Let $Z_{k}, k=0,1$, be known and fixed. The inverse problem is formulated as follows.

Inverse problem 1. Given the Weyl function $M(\lambda)$, construct the potential $q(x)$ and the coefficient $P_{00}$.

We note that if $p_{1}>0$, then in general $M(\lambda)$ does not uniquely determine all coefficients of the boundary condition. In Section 2 we study spectral properties of $L$. In Section 3 we provide an algorithm for the solution of the inverse problem considered. This algorithm is based on the reduction of our nonlinear inverse problem to the solution of the so-called main equation, which is a linear integral equation in a suitable Banach space. In this part of the paper we essentially use the results from [17]. The main result of the paper is Theorem 4 (see 
Section 4) where necessary and sufficient conditions are given for the solvability of Inverse problem 1.

\section{Spectral properties}

Denote by $W_{N}$ the set of functions $f(x), x \geq 0$ such that the functions $f^{(j)}(x), j=\overline{0, N-1}$ are absolutely continuous on $[0, T]$ for each fixed $T>0$, and $f^{(j)}(x) \in L(0, \infty), j=\overline{0, N}$. We shall say that $L \in \bar{W}_{N}$ if $q \in W_{N}$. We shall subsequently solve the inverse problem in the classes $\bar{W}_{N}$.

Let $S_{1}(x, \lambda), S_{2}(x, \lambda)$ and $\varphi(x, \lambda)$ be solutions of equation (1) under the initial conditions

$$
\begin{aligned}
S_{1}(0, \lambda) & =S_{2}^{\prime}(0, \lambda)=0, \quad S_{1}^{\prime}(0, \lambda)=S_{2}(0, \lambda)=1, \\
\varphi(0, \lambda) & =P_{1}(\lambda), \varphi^{\prime}(0, \lambda)=P_{0}(\lambda) .
\end{aligned}
$$

For each fixed $x$, and $v=0,1$, the functions $S_{j}^{(v)}(x, \lambda), j=1,2$, and $\varphi^{(v)}(x, \lambda)$ are entire in $\lambda$, and $\left\langle S_{2}(x, \lambda), S_{1}(x, \lambda)\right\rangle \equiv 1$, where $\langle y(x), z(x)\rangle:=y z^{\prime}-y^{\prime} z$ is the Wronskian of $y$ and $z$. Moreover,

$$
\left|S_{j}^{(v)}(x, \lambda)\right| \leq C|\rho|^{j+v-2}|\exp (-i \rho x)|, \quad x \geq 0, \quad v=0,1, \quad \rho \in \Omega .
$$

Clearly,

$$
\varphi(x, \lambda)=P_{1}(\lambda) S_{2}(x, \lambda)+P_{0}(\lambda) S_{1}(x, \lambda) .
$$

It is known [8] that equation (1) has a unique solution $e(x, \rho), \operatorname{Im} \rho \geq 0, x \geq 0$, with the properties:

(1) For each fixed $x \geq 0$, and $v=0,1$, the functions $e^{(v)}(x, \rho)$ are analytic for $\rho \in \Omega_{+}$, and are continuous for $\rho \in \Omega$.

(2) For $x \rightarrow \infty, v=0,1$, and each fixed $\delta>0$,

$$
e^{(v)}(x, \rho)=(i \rho)^{v} \exp (i \rho x)(1+o(1)),
$$

uniformly for $\rho \in \Omega,|\rho| \geq \delta$. For each $\rho \in \Omega_{+}, e(x, \rho) \in L_{2}(0, \infty)$. Moreover, $e(x, \rho)$ is the unique solution of (1) (up to a multiplicative constant) having this property.

(3) For $|\rho| \rightarrow \infty, \rho \in \Omega, v=0,1$,

$$
e^{(v)}(x, \rho)=(i \rho)^{v} \exp (i \rho x)\left(1+\sum_{s=1}^{N+1} \frac{\omega_{s v}(x)}{(i \rho)^{s}}+o\left(\frac{1}{\rho^{N+1}}\right)\right), \quad \omega_{1 v}(x):=-\frac{1}{2} \int_{x}^{\infty} q(t) d t,
$$

uniformly for $x \geq 0$.

The function $e(x, \rho)$ is called the Jost solution for $(1)$.

Denote

$$
\Delta(\rho):=P_{1}(\lambda) e^{\prime}(0, \rho)-P_{0}(\lambda) e(0, \rho)
$$


According to (5) and the definition of $\Phi(x, \lambda)$ and $M(\lambda)$ we have

$$
\Phi(x, \lambda)=\frac{e(x, \rho)}{\Delta(\rho)}, \quad M(\lambda)=\frac{e(0, \rho)}{\Delta(\rho)} .
$$

It follows from (4), (6)-(8) that for $\rho \in \Omega,|\rho|>\rho^{*}, v=0,1, x \geq 0$,

$$
\left|\Phi^{(v)}(x, \lambda)\right| \leq C|\rho|^{v-1}|\lambda|^{-p_{1}}|\exp (i \rho x)|, \quad\left|\varphi^{(v)}(x, \lambda)\right| \leq C|\rho|^{v}|\lambda|^{p_{1}}|\exp (-i \rho x)| .
$$

Denote

$$
\begin{aligned}
\Lambda & =\left\{\lambda=\rho^{2}: \rho \in \Omega, \Delta(\rho)=0\right\}, \\
\Lambda^{\prime} & =\left\{\lambda=\rho^{2}: \rho \in \Omega_{+}, \Delta(\rho)=0\right\}, \quad \Lambda^{\prime \prime}=\left\{\lambda=\rho^{2}: \rho \in \Omega_{0}, \rho \neq 0, \Delta(\rho)=0\right\} .
\end{aligned}
$$

Obviously, $\Lambda=\Lambda^{\prime} \cup \Lambda^{\prime \prime}$ is a bounded set, $\Lambda^{\prime \prime} \subset \Pi_{0}$, and $\Lambda^{\prime}$ is a bounded and at most countable set.

Theorem 1. The Weyl function $M(\lambda)$ is analytic in $\Pi_{+} \backslash \Lambda^{\prime}$ and continuous in $\Pi \backslash \Lambda$. The set of singularities of $M(\lambda)$ (as an analytic function) coincides with the set $\Lambda_{0}:=\Lambda_{+} \cup \Lambda$. For $|\rho| \rightarrow$ $\infty, \rho \in \Omega$,

$$
M(\lambda)=\frac{1}{(i \rho) \lambda^{p_{1}}}\left(1+\sum_{s=1}^{N+1} \frac{a_{s}}{(i \rho)^{s}}+o\left(\frac{1}{\rho^{N+1}}\right)\right)
$$

where $a_{1}=P_{00}$.

Theorem 1 follows from (6)-(8).

Definition 1. The set of singularities of the Weyl function $M(\lambda)$ is called the spectrum of $L$ (and is denoted by $\sigma(L)$ ). The values of the parameter $\lambda$, for which equation (1) has nontrivial solutions satisfying the conditions $U(y)=0, y(\infty)=0$ (i.e. $\lim _{x \rightarrow \infty} y(x)=0$ ), are called eigenvalues of $L$, and the corresponding solutions are called eigenfunctions of $L$.

The following theorem was proved in [17].

Theorem 2. L has no eigenvalues $\lambda>0$. Moreover, if $\lambda_{0}=\rho_{0}^{2}>0$ and $\Delta\left(\rho_{0}\right)=0$, then $\Delta\left(-\rho_{0}\right) \neq 0$. The set $\Lambda^{\prime}$ coincides with the set of non-zero eigenvalues of $L$.

Denote

$$
V(\lambda):=\frac{1}{2 \pi i}\left(M^{-}(\lambda)-M^{+}(\lambda)\right), \quad \lambda>0,
$$

where $M^{ \pm}(\lambda)=\lim _{z \rightarrow 0, R e z>0} M(\lambda \pm i z)$. Taking (10) into account we calculate

$$
V(\lambda)=\frac{1}{(\pi \rho) \lambda^{p_{1}}}\left(1+\sum_{s=1}^{N+1} \frac{v_{s}}{\rho^{s}}+o\left(\frac{1}{\rho^{N+1}}\right)\right),
$$


where $v_{2 s+1}=0, v_{2 s}=(-1)^{s} a_{2 s}$.

\section{The main equation}

For studying the inverse problem we agree that together with $L$ we consider a pair $\tilde{L}$ of the same form but with different potential $\tilde{q}$. If a certain symbol $\alpha$ denotes an object related to $L$, then the corresponding symbol $\tilde{\alpha}$ with tilde will denote the analogous object related to $\tilde{L}$, and $\hat{\alpha}:=\alpha-\tilde{\alpha}$. Then $Z_{k}=\tilde{Z}_{k}, k=0,1$.

Let $M(\lambda)$ be the Weyl function for $L$. Using (10) we find $P_{00}=a_{1}$, and construct $P_{k}(\lambda), k=$ 0,1 , by (3) where $P_{10}=1$. We choose a model pair $\tilde{L}=L(\tilde{q}, U)$ such that $\tilde{q} \in W_{N}, P_{k}(\lambda)=$ $\tilde{P}_{k}(\lambda), k=0,1$, and arbitrary in the rest. For example, one can take $\tilde{q}(x) \equiv 0$. In the $\lambda$ - plane we consider the contour $\gamma=\gamma^{\prime} \cup \gamma^{\prime \prime}$ (with counterclockwise circuit), where $\gamma^{\prime}$ is a bounded closed contour encircling the set $\Lambda \cup \tilde{\Lambda} \cup\{0\}$, and $\gamma^{\prime \prime}$ is the two-sided cut along the arc $\{\lambda: \lambda>$ $\left.0, \lambda \notin i n t \gamma^{\prime}\right\}$. It is proved in [17] that the following relation holds

$$
\tilde{\varphi}(x, \lambda)=\varphi(x, \lambda)+\frac{1}{2 \pi i} \int_{\gamma} \tilde{A}(x, \lambda, \mu) \varphi(x, \mu) d \mu,
$$

where

$$
\tilde{A}(x, \lambda, \mu)=\tilde{D}(x, \lambda, \mu) \frac{\hat{M}(\mu)}{P_{1}(\mu)}, \quad \tilde{D}(x, \lambda, \mu):=\frac{\langle\tilde{\varphi}(x, \lambda), \tilde{\varphi}(x, \mu)\rangle}{(\lambda-\mu)} .
$$

Moreover, for $\lambda, \mu \in \gamma, \lambda=\rho^{2}, \mu=\theta^{2}$,

$$
|\varphi(x, \lambda)|,|\tilde{\varphi}(x, \lambda)| \leq C|\lambda|^{p_{1}}, \quad|\tilde{A}(x, \lambda, \mu)| \leq \frac{C|\lambda|^{p_{1}}|\hat{M}(\mu)|}{|\rho-\chi \theta|+1},
$$

where $\chi:=\operatorname{sign}(\operatorname{Re} \rho \operatorname{Re} \theta)$. The integral in (12) converge absolutely for each $\lambda \in \gamma$ and $x \geq 0$. Analogously to (12) one can obtain the relation

$$
\tilde{\Phi}(x, \lambda)=\Phi(x, \lambda)+\frac{1}{2 \pi i} \int_{\gamma} \tilde{a}(x, \lambda, \mu) \varphi(x, \mu) d \mu, \quad \lambda \in J_{\gamma},
$$

where $J_{\gamma}=\left\{\lambda: \lambda \notin \gamma \cup\right.$ int $\left.\gamma^{\prime}\right\}$, and

$$
\tilde{a}(x, \lambda, \mu)=\frac{\langle\tilde{\Phi}(x, \lambda), \tilde{\varphi}(x, \mu)\rangle}{\lambda-\mu} \cdot \frac{\hat{M}(\mu)}{P_{1}(\mu)} .
$$

Relation (12) can be considered as a linear integral equation with respect to $\varphi(x, \lambda)$ with $x$ as a parameter. Equation (12) is called the main equation of the inverse problem.

Let us consider the Banach space $C(\gamma)$ of continuous bounded functions $z(\lambda), \lambda \in \gamma$, with the norm $\|z\|=\sup _{\lambda \in \gamma}|z(\lambda)|$. Denote by $C^{\prime}(\gamma)$ the set of functions $f(\lambda)$ such that $f(\lambda)|\lambda|^{-p_{1}} \in$ $C(\gamma)$. The following theorem was proved in [17]. 
Theorem 3. For each fixed $x \geq 0$, the main equation (12) has a unique solution $\varphi(x, \lambda) \in C^{\prime}(\gamma)$.

Denote

$$
\varepsilon_{0}(x)=\frac{1}{2 \pi i} \int_{\gamma} \tilde{\varphi}(x, \mu) \varphi(x, \mu) \frac{\hat{M}(\mu)}{P_{1}(\mu)} d \mu .
$$

Lemma 1. The following relation holds

$$
q(x)=\tilde{q}(x)+\varepsilon(x)
$$

where $\varepsilon(x)=-2 \varepsilon_{0}^{\prime}(x)$.

Proof. In order to simplify calculations we will assume in the sequel that $\hat{V}(\lambda) \lambda^{p_{1}+1 / 2} \in L\left(\lambda^{*}\right.$, $\infty$ ), for sufficiently large $\lambda^{*}$. For example, it is always true if $N>1$ and $\hat{v}_{2}=0$. Since

$$
\frac{d}{d x}\langle\tilde{\varphi}(x, \lambda), \tilde{\varphi}(x, \mu)\rangle=(\lambda-\mu) \tilde{\varphi}(x, \lambda) \tilde{\varphi}(x, \mu),
$$

it follows that

$$
\tilde{D}^{\prime}(x, \lambda, \mu)=\tilde{\varphi}(x, \lambda) \tilde{\varphi}(x, \mu) .
$$

Differentiating (12) twice with respect to $x$ and using (16) and (14) we get

$$
\begin{aligned}
\tilde{\varphi}^{\prime}(x, \lambda)-\varepsilon_{0}(x) \tilde{\varphi}(x, \lambda)= & \varphi^{\prime}(x, \lambda)+\frac{1}{2 \pi i} \int_{\gamma} \tilde{A}(x, \lambda, \mu) \varphi^{\prime}(x, \mu) d \mu, \\
\tilde{\varphi}^{\prime \prime}(x, \lambda)= & \varphi^{\prime \prime}(x, \lambda)+\frac{1}{2 \pi i} \int_{\gamma} \tilde{A}(x, \lambda, \mu) \varphi^{\prime \prime}(x, \mu) d \mu \\
& +\frac{1}{2 \pi i} \int_{\gamma} 2 \tilde{\varphi}(x, \lambda) \tilde{\varphi}(x, \mu) \frac{\hat{M}(\mu)}{P_{1}(\mu)} \varphi^{\prime}(x, \mu) d \mu \\
& +\frac{1}{2 \pi i} \int_{\gamma}(\tilde{\varphi}(x, \lambda) \tilde{\varphi}(x, \mu))^{\prime} \frac{\hat{M}(\mu)}{P_{1}(\mu)} \varphi(x, \mu) d \mu .
\end{aligned}
$$

In (18) we replace the second derivatives using equation (1), and then we replace $\varphi(x, \lambda)$ using (12). This yields

$$
\begin{aligned}
\tilde{q}(x) \tilde{\varphi}(x, \lambda)= & q(x) \tilde{\varphi}(x, \lambda)+\frac{1}{2 \pi i} \int_{\gamma}\langle\tilde{\varphi}(x, \lambda), \tilde{\varphi}(x, \mu)\rangle \frac{\hat{M}(\mu)}{P_{1}(\mu)} \varphi(x, \mu) d \mu \\
& +\frac{1}{2 \pi i} \int_{\gamma} 2 \tilde{\varphi}(x, \lambda) \tilde{\varphi}(x, \mu) \frac{\hat{M}(\mu)}{P_{1}(\mu)} \varphi^{\prime}(x, \mu) d \mu+\frac{1}{2 \pi i} \int_{\gamma}(\tilde{\varphi}(x, \lambda) \tilde{\varphi}(x, \mu))^{\prime} \frac{\hat{M}(\mu)}{P_{1}(\mu)} \varphi(x, \mu) d \mu .
\end{aligned}
$$

After canceling terms with $\tilde{\varphi}^{\prime}(x, \lambda)$ we arrive at (15).

Thus, we obtain the following algorithm for the solution of Inverse problem 1.

Algorithm 1. Let the function $M(\lambda)$ be given. Then

(1) Construct $P_{00}$ and $P_{k}(\lambda), k=0,1$. 
(3) Find $\varphi(x, \lambda)$ by solving equation (12).

(4) Construct $q(x)$ via (15).

This algorithm is similar to the corresponding algorithm from [17], but here we use another formula for calculating $q(x)$. This formula is more effective for numerical analysis (see [18]).

Lemma 2. The following relations hold

$$
\begin{aligned}
\hat{M}(\lambda) & =\frac{1}{2 \pi i} \int_{\gamma} \frac{\hat{M}(\mu)}{\lambda-\mu} d \mu, \quad \lambda \in J_{\gamma}, \\
P_{k}(\lambda) \hat{M}(\lambda) & =\frac{1}{2 \pi i} \int_{\gamma} \frac{P_{k}(\mu) \hat{M}(\mu)}{\lambda-\mu} d \mu, \quad \lambda \in J_{\gamma} .
\end{aligned}
$$

Proof. Consider the contour $\gamma_{R}^{0}=(\gamma \cap\{\lambda:|\lambda| \leq R\}) \cup\{\lambda:|\lambda|=R\}$ (with clockwise circuit). By Cauchy's integral formula

$$
\hat{M}(\lambda)=\frac{1}{2 \pi i} \int_{\gamma_{R}^{0}} \frac{\hat{M}(\mu)}{\lambda-\mu} d \mu, \quad \lambda \in i n t \gamma_{R}^{0} .
$$

By virtue of (10), $|\hat{M}(\lambda)| \leq C|\lambda|^{-p_{1}-1},|\lambda| \rightarrow \infty$, and consequently,

$$
\lim _{R \rightarrow \infty} \frac{1}{2 \pi i} \int_{|\mu|=R} \frac{\hat{M}(\mu)}{\lambda-\mu} d \mu=0
$$

Together with (21) this yields (19). Relation (20) is proved similarly.

Lemma 3. The following relations hold

$$
\begin{aligned}
& \frac{1}{2 \pi i} \int_{\gamma} \tilde{A}(0, \lambda, \mu) \varphi^{(v)}(0, \mu) d \mu=0, \quad v=0,1, \\
& \frac{1}{2 \pi i} \int_{\gamma} P_{1}(\mu) \hat{M}(\mu) d \mu=0 .
\end{aligned}
$$

Proof. Since

$$
\tilde{A}(x, \lambda, \mu)=\frac{\langle\tilde{\varphi}(x, \lambda), \tilde{\varphi}(x, \mu)\rangle}{(\lambda-\mu)} \cdot \frac{\hat{M}(\mu)}{P_{1}(\mu)}, \quad \tilde{\varphi}(0, \lambda)=P_{1}(\lambda), \quad \tilde{\varphi}^{\prime}(0, \lambda)=P_{0}(\lambda),
$$

it follows that

$$
\frac{1}{2 \pi i} \int_{\gamma} \tilde{A}(0, \lambda, \mu) \varphi(0, \mu) d \mu=P_{1}(\lambda) \frac{1}{2 \pi i} \int_{\gamma} \frac{P_{0}(\mu) \hat{M}(\mu)}{\lambda-\mu} d \mu-P_{0}(\lambda) \frac{1}{2 \pi i} \int_{\gamma} \frac{P_{1}(\mu) \hat{M}(\mu)}{\lambda-\mu} d \mu .
$$

Using (20) we calculate

$$
\frac{1}{2 \pi i} \int_{\gamma} \tilde{A}(0, \lambda, \mu) \varphi(0, \mu) d \mu=P_{1}(\lambda) P_{0}(\lambda) \hat{M}(\lambda)-P_{0}(\lambda) P_{1}(\lambda) \hat{M}(\lambda)=0,
$$


i.e. (22) is proved for $v=0$. For $v=1$, arguments are similar.

Put $x=0$ in (14) and (17). Then

$$
\varepsilon_{0}(0)=\frac{1}{2 \pi i} \int_{\gamma} P_{1}(\mu) \hat{M}(\mu) d \mu .
$$

Since $\tilde{\varphi}^{\prime}(0, \lambda)=\varphi^{\prime}(0, \lambda)=P_{0}(\lambda)$, it follows from (17) and (22) that $\varepsilon_{0}(0)=0$, i.e. (23) is proved.

\section{Necessary and sufficient conditions}

Let us now formulate necessary and sufficient conditions for the solvability of Inverse problem 1. Denote in the sequel by $V_{N}$ the set of functions $M(\lambda)$ such that

(i) the functions $M(\lambda)$ are analytic in $\Pi_{+}$with the exception of an at most countable bounded set $\Lambda^{\prime}$ of poles, and are continuous in $\Pi$ with the exception of bounded set $\Lambda$ (in general, $\Lambda$ and $\Lambda^{\prime}$ are different for each function $M(\lambda)$ );

(ii) for $|\lambda| \rightarrow \infty$, (10) holds, where $a_{s}$ are complex numbers.

Since $Z_{k}, k=0,1$, are known, we construct $P_{k}(\lambda)$ by (3), where $P_{10}=1, P_{00}=a_{1}$.

Theorem 4. For a function $M(\lambda) \in V_{N}$ to be the Weyl function for a certain $L \in \bar{W}_{N}$, it is necessary and sufficient that the following conditions hold:

(1) Relation(23) holds;

(2) (Condition S) For each fixed $x \geq 0$, equation (12) has a unique solution $\varphi(x, \lambda) \in C^{\prime}(\gamma)$;

(3) $\varepsilon_{0}(x) \in W_{N+1}$, where the function $\varepsilon_{0}(x)$ is defined by (14).

Under these conditions $q(x)$ is constructed via (15).

The necessity part of Theorem 4 was proved above. We prove now the sufficiency. Let a function $M(\lambda) \in V_{N}$, satisfying the hypothesis of Theorem 4 , be given, and let $\varphi(x, \lambda)$ be the solution of the main equation (12). Then (12) gives us the analytic continuation of $\varphi(x, \lambda)$ to the whole $\lambda$-plane, and for each fixed $x \geq 0$, the function $\varphi(x, \lambda)$ is entire in $\lambda$ of order 1/2. Using Lemma 1.5.1 from [8], by the standard technique, one can show that the functions $\varphi^{(v)}(x, \lambda), v=0,1,2$, are absolutely continuous with respect to $x$ on compact sets, and

$$
\left|\varphi^{(v)}(x, \lambda)\right| \leq C|\rho|^{v}|\lambda|^{p_{1}} \exp (|\tau| x), \quad|\eta(x, \lambda)| \leq C|\lambda|^{p_{1}+1} \exp (|\tau| x),
$$

where $\eta(x, \lambda):=\ell \varphi(x, \lambda)-\lambda \varphi(x, \lambda)$. We construct the function $\Phi(x, \lambda)$ via (13), and $q(x)$ by (15). Thus, we have a pair $L=L(q, U)$. Obviously, $L \in \bar{W}_{N}$. 
Lemma 4. The following relations hold

$$
\ell \varphi(x, \lambda)=\lambda \varphi(x, \lambda), \quad \ell \Phi(x, \lambda)=\lambda \Phi(x, \lambda) .
$$

Proof. Differentiating (12) twice with respect to $x$, we obtain (17) and (18). It follows from (18) and (12) that

$$
\begin{aligned}
-\tilde{\varphi}^{\prime \prime}(x, \lambda)+q(x) \tilde{\varphi}(x, \lambda)= & \ell \varphi(x, \lambda)+\frac{1}{2 \pi i} \int_{\gamma} \tilde{A}(x, \lambda, \mu) \ell \varphi(x, \mu) d \mu \\
& +\frac{1}{2 \pi i} \int_{\gamma}\langle\tilde{\varphi}(x, \lambda), \tilde{\varphi}(x, \mu)\rangle \frac{\hat{M}(\mu)}{P_{1}(\mu)} \varphi(x, \mu) d \mu \\
& -2 \tilde{\varphi}(x, \lambda) \frac{1}{2 \pi i} \int_{\gamma}(\tilde{\varphi}(x, \lambda) \tilde{\varphi}(x, \mu))^{\prime} \frac{\hat{M}(\mu)}{P_{1}(\mu)} \varphi(x, \mu) d \mu .
\end{aligned}
$$

Taking (15) into account we get

$$
\begin{aligned}
\tilde{\ell} \tilde{\varphi}(x, \lambda)= & \ell \varphi(x, \lambda)+\frac{1}{2 \pi i} \int_{\gamma} \tilde{A}(x, \lambda, \mu) \ell \varphi(x, \mu) d \mu \\
& +\frac{1}{2 \pi i} \int_{\gamma}\langle\tilde{\varphi}(x, \lambda), \tilde{\varphi}(x, \mu)\rangle \frac{\hat{M}(\mu)}{P_{1}(\mu)} \varphi(x, \mu) d \mu .
\end{aligned}
$$

Using (13) we calculate similarly

$$
\begin{aligned}
\tilde{\Phi}^{\prime}(x, \lambda)-\varepsilon_{0}(x) \tilde{\Phi}(x, \lambda)= & \Phi^{\prime}(x, \lambda)+\frac{1}{2 \pi i} \int_{\gamma} \frac{\langle\tilde{\Phi}(x, \lambda), \tilde{\varphi}(x, \mu)\rangle}{\lambda-\mu} \cdot \frac{\hat{M}(\mu)}{P_{1}(\mu)} \varphi^{\prime}(x, \mu) d \mu, \\
\tilde{\ell} \tilde{\Phi}(x, \lambda)= & \ell \Phi(x, \lambda)+\frac{1}{2 \pi i} \int_{\gamma} \frac{\langle\tilde{\Phi}(x, \lambda), \tilde{\varphi}(x, \mu)\rangle}{\lambda-\mu} \cdot \frac{\hat{M}(\mu)}{P_{1}(\mu)} \ell \varphi(x, \mu) d \mu \\
& +\frac{1}{2 \pi i} \int_{\gamma}\langle\tilde{\Phi}(x, \lambda), \tilde{\varphi}(x, \mu)\rangle \frac{\hat{M}(\mu)}{P_{1}(\mu)} \varphi(x, \mu) d \mu .
\end{aligned}
$$

It follows from (25) that

$$
\lambda \tilde{\varphi}(x, \lambda)=\ell \varphi(x, \lambda)+\frac{1}{2 \pi i} \int_{\gamma} \tilde{A}(x, \lambda, \mu) \ell \varphi(x, \mu) d \mu+\frac{1}{2 \pi i} \int_{\gamma}(\lambda-\mu) \tilde{A}(x, \lambda, \mu) \varphi(x, \mu) d \mu .
$$

Taking (12) into account we deduce for a fixed $x \geq 0$,

$$
\eta(x, \lambda)+\frac{1}{2 \pi i} \int_{\gamma} \tilde{A}(x, \lambda, \mu) \eta(x, \mu) d \mu=0, \quad \lambda \in \gamma .
$$

According to (24) we have

$$
|\eta(x, \lambda)| \leq C|\lambda|^{p_{1}+1}, \quad \lambda \in \gamma .
$$

By virtue of (28) and (29),

$$
|\eta(x, \lambda)| \leq C|\lambda|^{p_{1}}\left(1+\int_{\lambda^{*}}^{\infty} \frac{\mu^{p_{1}+1}|\hat{V}(\mu)|}{|\rho-\theta|+1} d \mu\right), \quad \lambda \in \gamma, \theta>0, \operatorname{Re} \rho \geq 0 .
$$


Since

$$
\frac{\theta}{\rho(|\rho-\theta|+1)} \leq 1 \text { for } \theta, \rho \geq 1
$$

this yields

$$
|\eta(x, \lambda)| \leq C|\lambda|^{p_{1}+1 / 2}, \quad \lambda \in \gamma .
$$

Using (30) instead of (29) and repeating the preceding arguments we infer

$$
|\eta(x, \lambda)| \leq C|\lambda|^{p_{1}}, \quad \lambda \in \gamma .
$$

According to Condition S of Theorem 4, the homogeneous equation (28) has only the trivial solution $\eta(x, \lambda) \equiv 0$. Consequently,

$$
\ell \varphi(x, \lambda)=\lambda \varphi(x, \lambda)
$$

It follows from (27) and (31) that

$$
\begin{aligned}
\lambda \tilde{\Phi}(x, \lambda)= & \ell \Phi(x, \lambda)+\frac{1}{2 \pi i} \int_{\gamma} \frac{\langle\tilde{\Phi}(x, \lambda), \tilde{\varphi}(x, \mu)\rangle}{\lambda-\mu} \frac{\hat{M}(\mu)}{P_{1}(\mu)} \mu \varphi(x, \mu) d \mu \\
& +\frac{1}{2 \pi i} \int_{\gamma}(\lambda-\mu) \frac{\langle\tilde{\Phi}(x, \lambda), \tilde{\varphi}(x, \mu)\rangle}{\lambda-\mu} \frac{\hat{M}(\mu)}{P_{1}(\mu)} \varphi(x, \mu) d \mu .
\end{aligned}
$$

Together with (13) this yields $\ell \Phi(x, \lambda)=\lambda \Phi(x, \lambda)$.

Lemma 5. The following relations hold

$$
\begin{aligned}
\varphi(0, \lambda) & =P_{1}(\lambda), \varphi^{\prime}(0, \lambda)=P_{0}(\lambda), \\
U(\Phi) & =1, \quad \Phi(0, \lambda)=M(\lambda), \\
\Phi(x, \lambda) & =O(\exp (i \rho x)), x \rightarrow \infty .
\end{aligned}
$$

Proof. It follows from (24) that

$$
\left|\varphi^{(v)}(0, \lambda)\right| \leq C|\rho|^{v}|\lambda|^{p_{1}} .
$$

We recall that in the necessity it was proved that (22) is valid. Using (32), by the same arguments as in the proof of Lemma 3, one can get that (22) is valid also in the sufficiency.

Taking $x=0$ in (12) and using (22) we get

$$
\varphi(0, \lambda)=P_{1}(\lambda)
$$

It follows from (14), (23) and (33) that

$$
\varepsilon_{0}(0)=\frac{1}{2 \pi i} \int_{\gamma} P_{1}(\mu) \hat{M}(\mu) d \mu=0 .
$$


Taking $x=0$ in (17) and using (22) and (34) we get

$$
\varphi^{\prime}(0, \lambda)=P_{0}(\lambda)
$$

Using (13) and (33) we calculate

$$
\tilde{\Phi}(0, \lambda)=\Phi(0, \lambda)+\frac{1}{2 \pi i} \int_{\gamma} \tilde{a}(0, \lambda, \mu) P_{1}(\mu) d \mu,
$$

and consequently,

$$
\tilde{\Phi}(0, \lambda)=\Phi(0, \lambda)+\tilde{\Phi}(0, \lambda) \frac{1}{2 \pi i} \int_{\gamma} \frac{P_{0}(\mu) \hat{M}(\mu)}{\lambda-\mu} d \mu-\tilde{\Phi}^{\prime}(0, \lambda) \frac{1}{2 \pi i} \int_{\gamma} \frac{P_{1}(\mu) \hat{M}(\mu)}{\lambda-\mu} d \mu .
$$

By virtue of Lemma 2 and the relation $U(\tilde{\Phi})=1$, this yields

$$
\tilde{\Phi}(0, \lambda)=\Phi(0, \lambda)-\hat{M}(\lambda) .
$$

Similarly, using (26) and (33)-(35) we calculate

$$
P_{1}(\lambda) \tilde{\Phi}^{\prime}(0, \lambda)=P_{1}(\lambda) \Phi(0, \lambda)-P_{0}(\lambda) \hat{M}(\lambda) .
$$

Since $U(\tilde{\Phi})=1, \tilde{\Phi}(0, \lambda)=\tilde{M}(\lambda)$, it follows from (36) and (37) that $U(\Phi)=1, \Phi(0, \lambda)=M(\lambda)$.

Furthermore, let us rewrite (13) in the form

$$
\Phi(x, \lambda)=\tilde{\Phi}(x, \lambda)+\frac{1}{2 \pi i} \int_{\gamma} \frac{\tilde{\Phi}^{\prime}(x, \lambda) \tilde{\varphi}(x, \mu)-\tilde{\Phi}(x, \lambda) \tilde{\varphi}^{\prime}(x, \mu)}{\lambda-\mu} \cdot \frac{\hat{M}(\mu)}{P_{1}(\mu)} \varphi(x, \mu) d \mu,
$$

where $\lambda \in J_{\gamma}$. According to (9) and (10) we have

$$
\begin{aligned}
\left|\varphi^{(v)}(x, \lambda)\right|,\left|\tilde{\varphi}^{(v)}(x, \lambda)\right| & \leq C|\rho|^{v}|\lambda|^{p_{1}}, \quad \lambda \in \gamma, x \geq 0, v=0,1, \\
\left|\tilde{\Phi}^{(v)}(x, \lambda)\right| & \leq C|\rho|^{v-1}|\lambda|^{-p_{1}}|\exp (i \rho x)|, \quad \rho \in \Omega,|\rho|>\rho^{*}, v=0,1, x \geq 0, \\
\left|\hat{M}(\lambda)\left(P_{1}(\lambda)\right)^{-1}\right| & \leq C|\lambda|^{-2 p_{1}-1}, \quad \rho \in \Omega,|\rho|>\rho^{*} .
\end{aligned}
$$

Together with (38) this yields for a fixed $\lambda \in J_{\gamma}$ :

$$
|\Phi(x, \lambda) \exp (-i \rho x)| \leq C_{\lambda}\left(1+\int_{\lambda^{*}}^{\infty} \frac{d \mu}{\mu^{1 / 2}|\lambda-\mu|}\right),
$$

and consequently, $\Phi(x, \lambda)=O(\exp (i \rho x)), x \rightarrow \infty$.

Thus, $M(\lambda)$ is the Weyl function for the constructed pair $L(q(x), U)$, and Theorem 4 is proved.

\section{Acknowledgement}

This research was supported in part by Grants 10-01-00099 and 10-01-92001-NSC of Russian Foundation for Basic Research and Taiwan National Science Council. 


\section{References}

[1] L. Collatz, Eigenwertaufgaben mit technischen Anwendungen, Akad. Verlagsgesellschaft Geest \& Portig, Leipzig, 1963.

[2] R. Mennicken and M. Möller, Non-self-adjoint boundary value problems. North-Holland Mathematic Studies, vol. 192, Amsterdam, North-Holland, 2003.

[3] A. A. Shkalikov, Boundary problems for opdinary problems for differential equations with parameter in the boundary conditions, J. Sov. Math., 33 (1986), 1311-1342; translation from Tr. Semin. im. I.G. Petrovskogo, 9 (1983), 190-229.

[4] Ch. Tretter, Boundary eigenvalue problems with differential equations $N \eta=\lambda P \eta$ with $\lambda$ - polynomial boundary conditions, J. Differ. Equ., 170 (2001), 408-471.

[5] V. A. Marchenko, Sturm-Liouville operators and their applications, Naukova Dumka, Kiev, 1977; English transl., Birkhäuser, 1986.

[6] B. M. Levitan, Inverse Sturm-Liouville Problems, Nauka, Moscow, 1984 (Russian); English transl., VNU Sci.Press, Utrecht, 1987.

[7] J. Pöschel and E. Trubowitz, Inverse Spectral Theory, New York, Academic Press, 1987.

[8] G. Freiling and V. A. Yurko, Inverse Sturm-Liouville Problems and their Applications. NOVA Science Publishers, New York, 2001.

[9] V. A. Yurko, Method of Spectral Mappings in the Inverse Problem Theory, Inverse and Ill-posed Problems Series. VSP, Utrecht, 2002.

[10] A. G. Ramm, Inverse problems. Mathematical and Analytical Techniques with Applications to Engineering. Springer, New York, 2005.

[11] P. J. Browne and B. D. Sleeman, A uniqueness theorem for inverse eigenparameter dependent Sturm-Liouville problems, Inverse Problems, 13 (1997), 1453-1462.

[12] M. V. Chugunova, Inverse spectral problem for the Sturm-Liouville operator with eigenvalue parameter dependent boundary conditions, Oper. Theory: Advan. Appl., 123, Birkhauser, Basel, 2001, 187-94.

[13] P. A. Binding, P. J. Browne and B. A. Watson, Sturm-Liouville problems with boundary conditions rationally dependent on the eigenparameter, II. J. Comp. Appl. Math., 148 (2002), 147-168.

[14] P. A. Binding, P. J. Browne and B. A. Watson, Equivalence of inverse Sturm-Liouville problems with boundary conditions rationally dependent on the eigenparameter, J. Math. Anal. Appl., 291 (2004), 246-261.

[15] N. J. Guliyev, Inverse eigenvalue problems for Sturm-Liouville equations with spectral parameter linearly contained in one of the boundary condition, Inverse Problems, 21 (2005), 1315-1330.

[16] G. Freiling and V. A. Yurko, Inverse problems for Sturm-Liouville equations with boundary conditions polynomially dependent on the spectral parameter, Inverse Problems, 26(2010), 055003, 17pp.

[17] Yurko V.A. Recovering non-self-adjoint Sturm-Liouville pencils on the half-line. Schriftenreiche des Fachbereichs Mathematik, SM-DU-726, Universitaet Duisburg-Essen, 2011, 10pp.

[18] M. Ignatiev and V. A. Yurko, Numerical methods for solving inverse Sturm-Liouville problems, Results in Math., 52 (2008), (2008), 63-74.

Department of Mathematics, Saratov University, Astrakhanskaya 83, Saratov 410012, Russia.

E-mail: yurkova@info.sgu.ru 